\title{
Local Stabilization for Multiple Input-Delay Systems subject to Saturating Actuators: The Continuous-time Case
}

\author{
Yonggang Chen, Zidong Wang, Bo Shen, and Qing-Long Han
}

\begin{abstract}
In this paper, the local stabilization problem is dealt with for a class of continuous-time multiple input-delay systems subject to saturating actuators. Using the generalized sector conditions and the piecewise Lyapunov-Krasovskii functional, and carrying out rigorous mathematical deduction, a sufficient condition is established under which the closed-loop dynamics is exponentially stable for admissible initial conditions. Subsequently, the explicit characterization of controller gains is obtained in terms of the solvability of linear matrix inequalities. The special cases concerning the constant and single delays are also discussed. Moreover, optimization problems are proposed to maximize the estimate of the domain of attraction. Finally, two simulation examples are given to show the effectiveness and advantages of the obtained results.
\end{abstract}

Index Terms-Continuous-time systems, local stabilization, control systems, multiple input delays, saturating actuators.

\section{INTRODUCTION}

In the past several decades, time-delay systems have gained considerable research attention from both communities of dynamical system and control engineering. In general, the motivation for the ever-increasing research interest is twofold: 1) many control systems intrinsically contain time delays in the state, the input or the output [3], [5], [7], [17], [18], [23], [25]; and 2) some strategies and phenomena can be modeled as time delays with examples including sampling control and packet dropouts [5], [26], [28]. For the stability analysis of time-delay systems, it has been acknowledged that the Lyapunov-Krasovskii (L-K) approach plays a vitally important role that enables the use of the linear matrix inequalities (LMIs), thereby facilitating the control design. Some important results under the L-K approach can be found in [5], [9], [16], [19], [21], [29]-[31].

The saturation phenomenon is frequently encountered in feedback control systems owing to the magnitude/rate constraints of physical actuators. Over the past two decades, the analysis/synthesis issue for control systems subject to saturating actuators has become an active topic of research in the control community [4], [8], [10]-[12], [22], [32]. In general, the existing results concerning the saturated control systems can be classified into two categories, i.e., the semiglobal/global case [12], [32] and the local case [4], [8], [10], [11], [22]. For the semi-global/global design, the main approaches include

This work was supported in part by the National Natural Science Foundation of China under Grants 61773156, 61873148, and 61933007, the Natural Science Foundation of Henan Province of China under Grant 202300410159, the Program for Science and Technology Innovation Talents in the Universities of Henan Province of China under Grant 19HASTIT028, the Royal Society of the U.K., and in part by the Alexander von Humboldt Foundation of Germany. (Corresponding author: Zidong Wang)

Y. Chen is with the School of Mathematical Sciences, Henan Institute of Science and Technology, Xinxiang 453003, China. (E-mail: happycygzmd@tom.com).

Z. Wang is with the College of Electrical Engineering and Automation, Shandong University of Science and Technology, Qingdao 266590, China, and is also with the Department of Computer Science, Brunel University London, Uxbridge, Middlesex, UB8 3PH, United Kingdom. (Email: Zidong.Wang@brunel.ac.uk).

B. Shen is with the College of Information Science and Technology, Donghua University, Shanghai 201620, China. (Email: bo.shen@dhu.edu.cn).

Q.-L. Han is with the School of Software and Electrical Engineering, Swinburne University of Technology, Melbourne, VIC 3122, Australia. (Email: qhan@swin.edu.au). the pole placement, the parametric Riccati/Lyapunov equation and the gain scheduling techniques [12], [32]. For the local analysis and synthesis, the polytopic models and the generalized sector conditions are the most popular techniques in dealing with saturations [8], [22].

In practical feedback control systems, it is often the case that actuator saturations and time delays coexist. Therefore, much effort has been devoted to the control synthesis for time-delay systems subject to actuator saturations in the past decade or so, see e.g. [1], [2], [6], [13]-[15], [27]. For example, in [1], [2], delay-dependent polytopic models have been explored to address the local stabilization problem for state delay systems with actuator saturations. Taking the first-delay interval into account, the local stabilization problem has been studied in [14] for input delay systems under actuator saturations. It should be pointed out that most existing literature has mainly focused on the case of a single delay. Considering that large-scale systems/networks might have multiple input delays, the semi-global stabilization problem has been sufficiently investigated in [33], [34] for linear systems with actuator saturations and multiple input delays. Nevertheless, it is worth mentioning that the semi-global stabilization scheme developed in [33], [34] cannot be directly applied to the case when the open-loop system is exponentially unstable.

Inspired by the above discussions, this paper takes one substantial step further by looking into the local stabilization problem for multiple input-delay systems with actuator saturations where the open-loop systems are allowed to be exponentially unstable. As in [3], [14], we redefine the initial conditions and estimate the solution bounds within initial time-intervals via the delay-dependent L-K approach. However, the main results of this paper are by no means a simple generalization of the existing ones (involving a single input delay) such as [14]. In fact, for feedback systems with multiple input delays, there exist multiple time-intervals within which the closed-loop dynamics are different. Therefore, the analysis approach proposed in [14] is unfortunately inapplicable. The main contributions of the paper are summarized as follows. 1) A piecewise L-K functional is constructed under which the exponential stability analysis of the closed-loop systems can be performed with certain flexibility. 2) By further incorporating the modified sector conditions, the local exponential stabilization conditions are established for a class of saturated control systems with multiple input delays.

Notation: The superscript " $T$ " stands for the transpose of a matrix. $P>0(\geq 0)$ denotes that $P$ is a real, symmetric, and positive definite (positive semi-definite) matrix. $\mathbb{R}^{n}$ is the $n$-dimensional Euclidean space. $u_{(l)}$ is the $l$-th element of the vector $u$, and $G_{(l)}$ denotes the $l$-th row of the matrix $G$. $\otimes$ is the Kronecker product. The symmetric terms in a symmetric matrix are denoted by $* . x_{t} \triangleq x(t+\theta), \theta \in$ $[-h, 0] . \operatorname{He}(\mathbb{A}) \triangleq \mathbb{A}+\mathbb{A}^{T} . \mathbf{I}[a, b] \triangleq\{a, a+1, \ldots, b\}$.

\section{PROBLEM FORMULATION}

Let us address the following nonlinear system subject to multiple time-varying input delays and saturating actuators:

$$
\dot{x}(t)=A x(t)+f(x(t))+\sum_{i=1}^{r} B_{i} \operatorname{sat}\left(u_{i}\left(t-\tau_{i}(t)\right)\right),
$$




$$
x(\theta)=x_{0}, \theta \in[-h, 0], h=\max _{1 \leq i \leq r}\left\{h_{i}\right\}
$$

where $x(t) \in \mathbb{R}^{n}$ denotes the system state; $u_{i}(t) \in \mathbb{R}^{m}(i \in \mathbf{I}[1, r])$ are control inputs with $u_{i}(t)=0$ for $t<0 ; A \in \mathbb{R}^{n \times n}$ and $B_{i} \in \mathbb{R}^{n \times m}(i \in \mathbf{I}[1, r])$ are known constant matrices; $\tau_{i}(t) \in\left[0, h_{i}\right]$ $(i \in \mathbf{I}[1, r])$ denote the time-varying input delays with known upperbounds $h_{i}>0 ; \operatorname{sat}\left(u_{i}\right)=\left[\operatorname{sat}\left(u_{i(1)}\right) \quad \operatorname{sat}\left(u_{i(2)}\right) \cdots \operatorname{sat}\left(u_{i(m)}\right)\right]^{T}$ $(i \in \mathbf{I}[1, r])$ are standard saturation functions with $\operatorname{sat}\left(u_{i(l)}\right)=$ $\operatorname{sgn}\left(u_{i(l)}\right) \min \left\{\left|u_{i(l)}\right|, \bar{u}_{i(l)}\right\}\left(\bar{u}_{i(l)}>0, l \in \mathbf{I}[1, m]\right) ; x_{0} \in \mathbb{R}^{n}$ is the initial condition; $f(x) \in \mathbb{R}^{n}$ is a continuous vector-valued function that satisfies $f(0)=0$ and the following sector-bounded condition:

$$
\left.f^{T}(x)\right)[f(x)-F x] \leq 0
$$

where $F \in \mathbb{R}^{n \times n}$ is a known real constant matrix.

Remark 1: As in literature [3], [14], we assume that $u_{i}(t)=0(i \in$ $\mathbf{I}[1, r])$ for $t<0$. It is worth mentioning that this control setting is often encountered in many control systems subject to actuator delays and transmission delays. Note that the solutions of the system (1) are independent on the values of $x(t)$ for $t<0$. Therefore, as with [3], [14], we define the initial condition as $x(t)=x_{0}$ within the interval $[-h, 0]$ for analyzing the stability by means of the L-K approach.

In this paper, we employ the following state feedback controllers:

$$
u_{i}(t)=K_{i} x(t), i \in \mathbf{I}[1, r], t \geq 0
$$

where $K_{i} \in \mathbb{R}^{m \times n}(i \in \mathbf{I}[1, r])$ are the controller gains.

Assumption 1: For every $\tau_{i}(t)(i \in \mathbf{I}[1, r])$, there exists a unique scalar $t_{i}^{*} \leq h_{i}$ such that $t-\tau_{i}(t)<0$ for $t<t_{i}^{*}$ and $t-\tau_{i}(t) \geq 0$ for $t \geq t_{i}^{*}$ [14]. Moreover, the scalars $t_{i}^{*}(i \in \mathbf{I}[1, r])$ satisfy

$$
0 \triangleq t_{0}^{*}<t_{1}^{*}<t_{2}^{*}<\cdots<t_{r}^{*}<h_{r}
$$

Denote $\psi(u) \triangleq u-\operatorname{sat}(u)$ and $t_{r+1}^{*} \triangleq+\infty$. Then, by applying (1) and (4), one has the following closed-loop system:

$$
\begin{aligned}
\dot{x}(t)= & A x(t)+f(x(t)), t \in\left[t_{0}^{*}, t_{1}^{*}\right), \\
\dot{x}(t)= & A x(t)+f(x(t))+\sum_{j=1}^{i} B_{j}\left[K_{j} x\left(t-\tau_{j}(t)\right)\right. \\
& \left.-\psi\left(u_{j}\left(t-\tau_{j}(t)\right)\right)\right], t \in\left[t_{i}^{*}, t_{i+1}^{*}\right), i \in \mathbf{I}[1, r] .
\end{aligned}
$$

Remark 2: In Assumption 1, the existence and uniqueness of the scalars $t_{i}^{*}(i \in \mathbf{I}[1, r])$ can be ensured if $\tau_{i}(t)(i \in \mathbf{I}[1, r])$ are slowlyvarying with $\dot{\tau}_{i}(t)<1$. The existence and uniqueness of $t_{i}^{*}$ are also guaranteed in networked control systems, where the modeled delays are piecewise-continuous and do not grow at the sampling instants [3], [14]. In addition, it is worth pointing out that, a somewhat strong requirement (5) is imposed for convenience of subsequent analysis.

Next, a lemma is introduced to deal with the dead-zone nonlinearities $\psi\left(u_{i}\right)(i \in \mathbf{I}[1, r])$ induced in the closed-loop system (7).

Lemma 1: [22] Let the vectors $u \in \mathbb{R}^{m}$ and $v \in \mathbb{R}^{m}$ be given. If $\left|u_{(l)}-v_{(l)}\right| \leq \bar{u}_{(l)}(l \in \mathbf{I}[1, m])$, then for any positive diagonal matrix $H \in \mathbb{R}^{m \times m}$, the following inequality holds:

$$
\psi^{T}(u) H[\psi(u)-v] \leq 0 .
$$

Denote $w \triangleq u-v$. It is obvious from Lemma 1 that, if $\left|w_{(l)}\right| \leq \bar{u}_{(l)}$ $(l \in \mathbf{I}[1, m])$, then the following sector condition is ensured:

$$
\psi^{T}(u) H[\psi(u)-u+w] \leq 0 .
$$

Let us introduce the vectors $w_{i j}=G_{i j} x \in \mathbb{R}^{m}(i \in \mathbf{I}[1, r]$, $j \in \mathbf{I}[1, i])$ where $G_{i j} \in \mathbb{R}^{m \times n}$. If the constraint conditions

$$
\begin{aligned}
& \left|w_{i j(l)}(t)\right|=\left|G_{i j(l)} x(t)\right| \leq \bar{u}_{j(l)}, t \in\left[t_{i}^{*}, t_{i+1}^{*}\right), \\
& i \in \mathbf{I}[1, r], \quad j \in \mathbf{I}[1, i], \quad l \in \mathbf{I}[1, m]
\end{aligned}
$$

are true, then for any positive diagonal matrices $H_{i j} \in \mathbb{R}^{m \times m}(i \in$ $\mathbf{I}[1, r], j \in \mathbf{I}[1, i])$, it follows from (8) that

$$
\begin{aligned}
& -2 \psi^{T}\left(u_{j}\left(t-\tau_{j}(t)\right)\right) H_{i j} \\
& \times\left[\psi\left(u_{j}\left(t-\tau_{j}(t)\right)\right)-K_{j} x\left(t-\tau_{j}(t)\right)+G_{i j} x(t)\right] \geq 0, \\
& \quad t \in\left[t_{i}^{*}, t_{i+1}^{*}\right), i \in \mathbf{I}[1, r], j \in \mathbf{I}[1, i] .
\end{aligned}
$$

Remark 3: To deal with the delayed dead-zone function $\psi(u(t-$ $\tau(t)))$, the delay-dependent sector condition has been employed in [5] (pp. 239). Compared with the sector condition used in [5], the sector conditions (10) depend on both the delayed states and the current state. Using the sector conditions (10), not only the relationships between $\psi\left(u_{j}\left(t-\tau_{j}(t)\right)\right)$ and $x\left(t-\tau_{j}(t)\right)(j \in \mathbf{I}[1, i])$ but also the relationships between $\psi\left(u_{j}\left(t-\tau_{j}(t)\right)\right)$ and $x(t)(j \in \mathbf{I}[1, i])$ are considered, which would be helpful in reducing the conservatism.

The main purpose of this paper is to design the state feedback controllers (4) such that the closed-loop system (6)-(7) is locally exponentially stable and, meanwhile, the estimate of the domain of attraction (DOA) is made as large as possible. Throughout this paper, the exponential stability means that the exponential convergence of the system trajectories are guaranteed from the instant $t_{r}^{*}$.

\section{MAIN RESULTS}

For the exponential stability analysis of the system (6)-(7), we propose a piecewise $\mathrm{L}-\mathrm{K}$ functional described as

$$
V(t)=\left\{\begin{array}{l}
V_{k}(t), t \in\left[t_{k}^{*}, t_{k+1}^{*}\right), k \in \mathbf{I}[0, r-1], \\
V_{r}(t), t \in\left[t_{r}^{*},+\infty\right)
\end{array}\right.
$$

where

$$
\begin{aligned}
V_{k}(t)= & x^{T}(t) P_{k} x(t)+\sum_{\hat{j}=1}^{r}\left[\int_{t-h_{\hat{j}}}^{t} e^{\alpha_{k}(t-s)} x^{T}(s) Q_{k \hat{j}} x(s) \mathrm{d} s\right. \\
& \left.+h_{\hat{j}} \int_{-h_{\hat{j}}}^{0} \int_{t+\theta}^{t} e^{\alpha_{k}(t-s)} \dot{x}^{T}(s) Z_{k \hat{j}} \dot{x}(s) \mathrm{d} s \mathrm{~d} \theta\right], \\
V_{r}(t)= & x^{T}(t) P_{r} x(t)+\sum_{\hat{j}=1}^{r}\left[\int_{t-h_{\hat{j}}}^{t} e^{\alpha_{r}(s-t)} x^{T}(s) Q_{r \hat{j}} x(s) \mathrm{d} s\right. \\
& \left.+h_{\hat{j}} \int_{-h_{\hat{j}}}^{0} \int_{t+\theta}^{t} e^{\alpha_{r}(s-t)} \dot{x}^{T}(s) Z_{r \hat{j}} \dot{x}(s) \mathrm{d} s \mathrm{~d} \theta\right]
\end{aligned}
$$

with $P_{\hat{i}}>0, Q_{\hat{i} \hat{j}}>0, Z_{\hat{i} \hat{j}}>0, \alpha_{\hat{i}}>0, \hat{i} \in \mathbf{I}[0, r], \hat{j} \in \mathbf{I}[1, r]$.

For convenience of subsequent presentation, we denote

$$
\begin{aligned}
& \xi_{0}(t) \triangleq\left[\begin{array}{llll}
x^{T}(t) & x^{T}\left(t-h_{1}\right) & x^{T}\left(t-h_{2}\right) & \cdots
\end{array}\right. \\
& \left.x^{T}\left(t-h_{r}\right) \quad f^{T}(x(t)) \dot{x}^{T}(t)\right]^{T}, \\
& \xi_{i}(t) \triangleq\left[\begin{array}{llll}
x^{T}(t) & x^{T}\left(t-\tau_{1}(t)\right) & \cdots & x^{T}\left(t-\tau_{i}(t)\right)
\end{array}\right. \\
& x^{T}\left(t-h_{1}\right) \cdots x^{T}\left(t-h_{r}\right) \quad \psi^{T}\left(u_{1}\left(t-\tau_{1}(t)\right)\right) \\
& \left.\cdots \psi^{T}\left(u_{i}\left(t-\tau_{i}(t)\right)\right) \quad f^{T}(x(t)) \dot{x}^{T}(t)\right]^{T}, \\
& \hat{Q}_{s \hat{j}} \triangleq e^{\alpha_{s} h_{\hat{j}}} Q_{s \hat{j}}, \hat{Q}_{r \hat{j}} \triangleq e^{-\alpha_{r} h_{\hat{j}}} Q_{r \hat{j}}, \check{Q}_{s \hat{j}} \triangleq e^{\alpha_{s} h_{\hat{j}}} \bar{Q}_{s \hat{j}} \\
& \check{Q}_{r \hat{j}} \triangleq e^{-\alpha_{r} h_{\hat{j}}} \bar{Q}_{r \hat{j}}, \hat{Z}_{s \hat{j}} \triangleq Z_{s \hat{j}}, \hat{Z}_{r \hat{j}} \triangleq e^{-\alpha_{r} h_{\hat{j}}} Z_{r \hat{j}}, \\
& \check{Z}_{s \hat{j}} \triangleq \bar{Z}_{s \hat{j}}, \check{Z}_{r \hat{j}} \triangleq e^{-\alpha_{r} h_{\hat{j}}} \bar{Z}_{r \hat{j}}, \hat{M}_{s j} \triangleq M_{s j} \text {, } \\
& \hat{M}_{r j} \triangleq e^{-\alpha_{r} h_{\hat{j}}} M_{r j}, \check{M}_{s j} \triangleq \bar{M}_{s j}, \check{M}_{r j} \triangleq e^{-\alpha_{r} h_{\hat{j}}} \bar{M}_{r j} \text {, } \\
& \beta_{s} \triangleq e^{\alpha_{0}\left(t_{1}^{*}-t_{0}^{*}\right)+\alpha_{1}\left(t_{2}^{*}-t_{1}^{*}\right)+\cdots+\alpha_{s}\left(t_{s+1}^{*}-t_{s}^{*}\right)} \mu_{1} \cdots \mu_{s}, \\
& \beta_{r} \triangleq \mu_{r} \beta_{r-1}, s \in \mathbf{I}[1, r-1], j \in \mathbf{I}[1, i], \hat{j} \in \mathbf{I}[1, r] .
\end{aligned}
$$

Theorem 1: Let the scalars $\alpha_{\hat{i}}>0$ and $\mu_{i}>0$ be given. Assume that there exist $n \times n$ matrices $P_{\hat{i}}>0, Q_{\hat{i} \hat{j}}>0, Z_{\hat{i} \hat{j}}>0, R_{\hat{i}}, S_{\hat{i}}$, $M_{i j}, m \times n$ matrices $K_{i}, G_{i j}, m \times m$ diagonal matrices $H_{i j}>0$, 
and scalars $\varepsilon_{\hat{i}}>0, \hat{i} \in \mathbf{I}[0, r], i, \hat{j} \in \mathbf{I}[1, r], j \in \mathbf{I}[1, i]$, such that for $\forall i, \hat{j} \in \mathbf{I}[1, r], \forall j \in \mathbf{I}[1, i]$, the following matrix inequalities holds:

$$
\begin{aligned}
& {\left[\begin{array}{cc}
Z_{i j} & M_{i j} \\
M_{i j}^{T} & Z_{i j}
\end{array}\right] \triangleq \Lambda_{i j}>0,} \\
& {\left[\begin{array}{cccc}
\Omega_{11}^{0} & \Omega_{12}^{0} & \Omega_{13}^{0} & \Omega_{14}^{0} \\
* & \Omega_{22}^{0} & 0 & 0 \\
* & * & -2 \varepsilon_{0} I & S_{0}^{T} \\
* & * & * & \Omega_{44}^{0}
\end{array}\right] \triangleq \Omega_{0}<0,} \\
& {\left[\begin{array}{cccccc}
\Omega_{11}^{i} & \Omega_{12}^{i} & \Omega_{13}^{i} & \Omega_{14}^{i} & \Omega_{15}^{i} & \Omega_{16}^{i} \\
* & \Omega_{22}^{i} & \Omega_{23}^{i} & \Omega_{24}^{i} & 0 & \Omega_{26}^{i} \\
* & * & \Omega_{33}^{i} & 0 & 0 & 0 \\
* & * & * & \Omega_{44}^{i} & 0 & \Omega_{46}^{i} \\
* & * & * & * & -2 \varepsilon_{i} I & S_{i}^{T} \\
* & * & * & * & * & \Omega_{66}^{i}
\end{array}\right] \triangleq \Omega_{i}<0,} \\
& \left\{\begin{array}{l}
P_{i} \leq \mu_{i} P_{i-1}, e^{\left(\alpha_{s}-\alpha_{s-1}\right) h_{\hat{j}}} Q_{s \hat{j}} \leq \mu_{s} Q_{s-1, \hat{j}}, \\
e^{\left(\alpha_{s}-\alpha_{s-1}\right) h_{\hat{j}}} Z_{s \hat{j}} \leq \mu_{s} Z_{s-1, \hat{j}}, \forall s \in \mathbf{I}[1, r-1], \\
Q_{r \hat{j}} \leq \mu_{r} Q_{r-1, \hat{j}}, Z_{r \hat{j}} \leq \mu_{r} Z_{r-1, \hat{j}},
\end{array}\right. \\
& {\left[\begin{array}{cc}
\bar{u}_{j(l)}^{2} / \beta_{i} & G_{i j(l)} \\
G_{i j(l)}^{T} & P_{i}
\end{array}\right] \geq 0, \forall l \in \mathbf{I}[1, m]}
\end{aligned}
$$

where

$$
\begin{aligned}
& \Omega_{11}^{0} \triangleq \operatorname{He}\left(R_{0} A\right)-\alpha_{0} P_{0}+\Sigma_{\hat{j}=1}^{r}\left(Q_{0 \hat{j}}-Z_{0 \hat{j}}\right), \\
& \Omega_{12}^{0} \triangleq\left[\begin{array}{llll}
Z_{01} & Z_{02} & \cdots & Z_{0 r}
\end{array}\right], \Omega_{13}^{0} \triangleq R_{0}+\varepsilon_{0} F^{T} \text {, } \\
& \Omega_{22}^{0} \triangleq \operatorname{diag}\left\{-e^{\alpha_{0} h_{1}} Q_{01}-Z_{01}, \ldots,-e^{\alpha_{0} h_{r}} Q_{0 r}-Z_{0 r}\right\} \text {, } \\
& \Omega_{14}^{0} \triangleq P_{0}-R_{0}+A^{T} S_{0}^{T}, \Omega_{44}^{0} \triangleq \Sigma_{\hat{j}=1}^{r} h_{\hat{j}}^{2} Z_{0 \hat{j}}-\operatorname{He}\left(S_{0}\right), \\
& \Omega_{11}^{s} \triangleq \operatorname{He}\left(R_{s} A\right)-\alpha_{s} P_{s}+\Sigma_{\hat{j}=1}^{r}\left(Q_{s \hat{j}}-\hat{Z}_{s \hat{j}}\right), s \in \mathbf{I}[1, r-1], \\
& \Omega_{11}^{r} \triangleq \operatorname{He}\left(R_{r} A\right)+\alpha_{r} P_{r}+\sum_{\hat{j}=1}^{r}\left(Q_{r \hat{j}}-\hat{Z}_{r \hat{j}}\right), \\
& \Omega_{12}^{i} \triangleq\left[R_{i} B_{1} K_{1}+\hat{Z}_{i 1}-\hat{M}_{i 1} \cdots R_{i} B_{i} K_{i}+\hat{Z}_{i i}-\hat{M}_{i i}\right] \text {, } \\
& \Omega_{13}^{s} \triangleq\left[\begin{array}{llllll}
\hat{M}_{s 1} & \cdots & \hat{M}_{s s} & \hat{Z}_{s, s+1} & \cdots & \hat{Z}_{s r}
\end{array}\right], s \in \mathbf{I}[1, r-1], \\
& \Omega_{14}^{i} \triangleq\left[\begin{array}{lll}
-R_{i} B_{1}-G_{i 1}^{T} H_{i 1}^{T} & \cdots & \left.-R_{i} B_{i}-G_{i i}^{T} H_{i i}^{T}\right],
\end{array}\right. \\
& \Omega_{15}^{i} \triangleq R_{i}+\varepsilon_{i} F^{T}, \Omega_{16}^{i} \triangleq-R_{i}+A^{T} S_{i}^{T}+P_{i}, \\
& \Omega_{22}^{i} \triangleq \operatorname{diag}\left\{\operatorname{He}\left(\hat{M}_{i 1}\right)-2 \hat{Z}_{i 1}, \ldots, \operatorname{He}\left(\hat{M}_{i i}\right)-2 \hat{Z}_{i i}\right\} \text {, } \\
& \Omega_{23}^{i} \triangleq\left[\operatorname{diag}\left\{\hat{Z}_{i 1}-\hat{M}_{i 1}, \ldots, \hat{Z}_{i i}-\hat{M}_{i i}\right\} \quad 0_{i n \times(r-i) n}\right] \text {, } \\
& \Omega_{24}^{i} \triangleq \operatorname{diag}\left\{K_{1}^{T} H_{i 1}^{T}, \ldots, K_{i}^{T} H_{i i}^{T}\right\}, \Omega_{13}^{r} \triangleq\left[\begin{array}{lll}
\hat{M}_{r 1} & \cdots & \hat{M}_{r r}
\end{array}\right] \text {, } \\
& \Omega_{26}^{i} \triangleq\left[\begin{array}{lll}
B_{1} K_{1} & \cdots & B_{i} K_{i}
\end{array}\right]^{T} S_{i}^{T}, \Omega_{46}^{i} \triangleq-\left[B_{1} \cdots B_{i}\right]^{T} S_{i}^{T} \text {, } \\
& \Omega_{33}^{i} \triangleq \operatorname{diag}\left\{-\hat{Q}_{i 1}-\hat{Z}_{i 1}, \ldots,-\hat{Q}_{i r}-\hat{Z}_{i r}\right\} \text {, } \\
& \Omega_{44}^{i} \triangleq-2 \operatorname{diag}\left\{H_{i 1}, \ldots, H_{i i}\right\}, \Omega_{66}^{i} \triangleq \sum_{\hat{j}=1}^{r} h_{\hat{j}}^{2} Z_{i \hat{j}}-\operatorname{He}\left(S_{i}\right) .
\end{aligned}
$$

Then, for all initial conditions $x_{0}$ satisfying $V_{0}(0) \leq 1$, the closedloop system (6)-(7) is exponentially stable.

Proof: Noting $V_{\hat{i}}(t)(\hat{i} \in \mathbf{I}[0, r])$ denoted in (11), we have

$$
\begin{aligned}
\dot{V}_{k}(t) \leq & 2 x^{T}(t) P_{k} \dot{x}(t)+\sum_{\hat{j}=1}^{r}\left[x^{T}(t) Q_{k \hat{j}} x(t)-e^{\alpha_{k} h_{\hat{j}}}\right. \\
& \times x^{T}\left(t-h_{\hat{j}}\right) Q_{k \hat{j}} x\left(t-h_{\hat{j}}\right)+h_{\hat{j}}^{2} \dot{x}^{T}(t) Z_{k \hat{j}} \dot{x}(t) \\
& \left.-h_{\hat{j}} \int_{t-h_{\hat{j}}}^{t} \dot{x}^{T}(s) Z_{k \hat{j}} \dot{x}(s) \mathrm{d} s\right]-\alpha_{k} x^{T}(t) P_{k} x(t) \\
& +\alpha_{k} V_{k}(t), t \in\left[t_{k}^{*}, t_{k+1}^{*}\right), k \in \mathbf{I}[0, r-1], \\
\dot{V}_{r}(t) \leq & 2 x^{T}(t) P_{r} \dot{x}(t)+\sum_{\hat{j}=1}^{r}\left[x^{T}(t) Q_{r \hat{j}} x(t)-e^{-\alpha_{r} h_{\hat{j}}}\right. \\
& \times x^{T}\left(t-h_{\hat{j}}\right) Q_{r \hat{j}} x\left(t-h_{\hat{j}}\right)+h_{\hat{j}}^{2} \dot{x}^{T}(t) Z_{r \hat{j}} \dot{x}(t) \\
& \left.-e^{-\alpha_{r} h_{\hat{j}}} h_{\hat{j}} \int_{t-h_{\hat{j}}}^{t} \dot{x}^{T}(s) Z_{r \hat{j}} \dot{x}(s) \mathrm{d} s\right]
\end{aligned}
$$

$$
+\alpha_{r} x^{T}(t) P_{r} x(t)-\alpha_{r} V_{r}(t), t \in\left[t_{r}^{*},+\infty\right) .
$$

Using the Jensen's integral inequality [14], it follows that

$$
\begin{aligned}
& h_{\hat{j}} \int_{t-h_{\hat{j}}}^{t} \dot{x}^{T}(s) Z_{0 \hat{j}} \dot{x}(s) \mathrm{d} s \geq \zeta_{1 \hat{j}}^{T}(t) Z_{0 \hat{j}} \zeta_{1 \hat{j}}(t), \hat{j} \in \mathbf{I}[1, r], \\
& h_{j} \int_{t-h_{j}}^{t} \dot{x}^{T}(s) Z_{i j} \dot{x}(s) \mathrm{d} s \geq \frac{h_{j}}{\tau_{j}(t)} \zeta_{2 j}^{T}(t) Z_{i j} \zeta_{2 j}(t) \\
& \quad+\frac{h_{j}}{h_{j}-\tau_{j}(t)} \zeta_{3 j}^{T}(t) Z_{i j} \zeta_{3 j}(t), i \in \mathbf{I}[1, r], j \in \mathbf{I}[1, i], \\
& h_{j} \int_{t-h_{j}}^{t} \dot{x}^{T}(s) Z_{i j} \dot{x}(s) \mathrm{d} s \geq \zeta_{4 j}^{T}(t) Z_{i j} \zeta_{4 j}(t), \\
& \quad i \in \mathbf{I}[1, r], j \in \mathbf{I}[i+1, r]
\end{aligned}
$$

where $\zeta_{1 \hat{j}}(t) \triangleq x(t)-x\left(t-h_{\hat{j}}\right), \zeta_{2 j}(t) \triangleq x(t)-x\left(t-\tau_{j}(t)\right)$, $\zeta_{3 j}(t) \triangleq x\left(t-\tau_{j}(t)\right)-x\left(t-h_{j}\right)$ and $\zeta_{4 j}(t) \triangleq x(t)-x\left(t-h_{j}\right)$.

If there exist matrices $M_{i j} \in \mathbb{R}^{n \times n}(i \in \mathbf{I}[1, r], j \in \mathbf{I}[1, i])$ such that LMIs (12) hold, the inequalities (20) can be modified as [19]

$$
\begin{gathered}
h_{j} \int_{t-h_{j}}^{t} \dot{x}^{T}(s) Z_{i j} \dot{x}(s) \mathrm{d} s \geq\left[\begin{array}{l}
\zeta_{2 j}(t) \\
\zeta_{3 j}(t)
\end{array}\right]^{T} \Lambda_{i j}\left[\begin{array}{l}
\zeta_{2 j}(t) \\
\zeta_{3 j}(t)
\end{array}\right], \\
i \in \mathbf{I}[1, r], \quad j \in \mathbf{I}[1, i] .
\end{gathered}
$$

For any matrices $R_{\hat{i}} \in \mathbb{R}^{n \times n}$ and $S_{\hat{i}} \in \mathbb{R}^{n \times n}, \hat{i} \in \mathbf{I}[0, r]$, it can be seen from the system equations (6) and (7) that [14]

$$
\begin{aligned}
& 2[A x(t)+f(x(t))-\dot{x}(t)]^{T}\left[R_{0}^{T} x(t)+S_{0}^{T} \dot{x}(t)\right]=0, \\
& 2\left[A x(t)+f(x(t))+\eta_{i}(t)-\dot{x}(t)\right]^{T} \\
& \quad \times\left[R_{i}^{T} x(t)+S_{i}^{T} \dot{x}(t)\right]=0, \quad i \in \mathbf{I}[1, r]
\end{aligned}
$$

where $\eta_{i}(t) \triangleq \sum_{j=1}^{i} B_{j}\left[K_{j} x\left(t-\tau_{j}(t)\right)-\psi\left(u_{j}\left(t-\tau_{j}(t)\right)\right)\right]$. Also, for any scalars $\varepsilon_{\hat{i}}>0(\hat{i} \in \mathbf{I}[0, r])$, one has from (3) that

$$
\begin{aligned}
& \left.-2 \varepsilon_{0} f^{T}(x(t))\right)[f(x(t))-F x(t)] \geq 0, \\
& \left.-2 \varepsilon_{i} f^{T}(x(t))\right)[f(x(t))-F x(t)] \geq 0, \quad i \in \mathbf{I}[1, r] .
\end{aligned}
$$

Adding the left-hand sides of (23) and (25) to $\dot{V}_{0}(t)$ denoted in (17), and using the inequalities (19), it follows that

$$
\begin{aligned}
\dot{V}_{0}(t) \leq & \dot{V}_{0}(t)+2[A x(t)+f(x(t))-\dot{x}(t)]^{T} \\
& \left.\times\left[R_{0}^{T} x(t)+S_{0}^{T} \dot{x}(t)\right]-2 \varepsilon_{0} f^{T}(x(t))\right)[f(x(t))-F x(t)] \\
\leq & \xi_{0}^{T}(t) \Omega_{0} \xi_{0}(t)+\alpha_{0} V_{0}(t), t \in\left[t_{0}^{*}, t_{1}^{*}\right) .
\end{aligned}
$$

Adding the left-hand sides of (10), (24) and (26) to $\dot{V}_{i}(t)$ in (17), and using (22) for $j \in \mathbf{I}[1, i]$ and (21) for $j \in \mathbf{I}[i+1, r]$, we have

$$
\begin{aligned}
\dot{V}_{i}(t) \leq & \dot{V}_{i}(t)-2 \Sigma_{j=1}^{i} \psi^{T}\left(u_{j}\left(t-\tau_{j}(t)\right)\right) H_{i j} \\
& \times\left[\psi\left(u_{j}\left(t-\tau_{j}(t)\right)\right)-K_{j} x\left(t-\tau_{j}(t)\right)+G_{i j} x(t)\right] \\
& +2\left[A x(t)+f(x(t))+\eta_{i}(t)-\dot{x}(t)\right]^{T} \\
& \left.\times\left[R_{i}^{T} x(t)+S_{i}^{T} \dot{x}(t)\right]-2 \varepsilon_{i} f^{T}(x(t))\right)[f(x(t))-F x(t)] \\
\leq & \xi_{i}^{T}(t) \Omega_{i} \xi_{i}(t)+\alpha_{i} V_{i}(t), t \in\left[t_{i}^{*}, t_{i+1}^{*}\right), i \in \mathbf{I}[1, r-1] .
\end{aligned}
$$

Similarly, it follows from (10), (18), (22), (24) and (26) that

$$
\dot{V}_{r}(t) \leq \xi_{r}^{T}(t) \Omega_{r} \xi_{r}(t)-\alpha_{r} V_{r}(t), t \in\left[t_{r}^{*},+\infty\right) .
$$

For $\forall i \in \mathbf{I}[1, r], \forall j \in \mathbf{I}[1, i]$, if the matrix inequalities (12)-(14) are feasible, then one obtains from (27)-(29) that

$$
\begin{aligned}
& V_{0}(t) \leq e^{\alpha_{0}\left(t-t_{0}^{*}\right)} V_{0}\left(t_{0}^{*}\right), t \in\left[t_{0}^{*}, t_{1}^{*}\right), \\
& V_{i}(t) \leq e^{\alpha_{i}\left(t-t_{i}^{*}\right)} V_{i}\left(t_{i}^{*}\right), t \in\left[t_{i}^{*}, t_{i+1}^{*}\right), i \in \mathbf{I}[1, r-1], \\
& V_{r}(t) \leq e^{-\alpha_{r}\left(t-t_{r}^{*}\right)} V_{r}\left(t_{r}^{*}\right), t \in\left[t_{r}^{*},+\infty\right) .
\end{aligned}
$$


Also, it is seen from the matrix inequalities (15) that

$$
V_{i}(t) \leq \mu_{i} V_{i-1}(t), i \in \mathbf{I}[1, r] .
$$

Using the inequalities (30), (31) and (33), it can be inferred that

$$
\begin{aligned}
& V_{1}(t) \leq e^{\alpha_{1}\left(t-t_{1}^{*}\right)} V_{1}\left(t_{1}^{*}\right) \leq e^{\alpha_{1}\left(t-t_{1}^{*}\right)} \mu_{1} V_{0}\left(t_{1}^{*}\right) \\
& \quad \leq e^{\alpha_{0}\left(t_{1}^{*}-t_{0}^{*}\right)+\alpha_{1}\left(t-t_{1}^{*}\right)} \mu_{1} V_{0}(0) \leq \beta_{1} V_{0}(0), t \in\left[t_{1}^{*}, t_{2}^{*}\right)
\end{aligned}
$$

where the scalar $\beta_{1}$ is denoted above Theorem 1. Using the similar arguments, we can eventually obtain from (30)-(33) that

$$
\begin{aligned}
& V_{i}(t) \leq \beta_{i} V_{0}(0), t \in\left[t_{i}^{*}, t_{i+1}^{*}\right), i \in \mathbf{I}[1, r-1], \\
& V_{r}(t) \leq \beta_{r} e^{-\alpha_{r}\left(t-t_{r}^{*}\right)} V_{0}(0), t \in\left[t_{r}^{*},+\infty\right) .
\end{aligned}
$$

On the other hand, it follows from (11) that

$$
V_{i}(t)>x^{T}(t) P_{i} x(t), i \in \mathbf{I}[1, r], x_{t} \neq 0 .
$$

For $\forall i \in \mathbf{I}[1, r], \forall j \in \mathbf{I}[1, i]$, if the matrix inequalities (16) are satisfied, using Schur complement, it is seen that

$$
\begin{aligned}
& P_{i} \geq\left(\beta_{i} / \bar{u}_{j(l)}^{2}\right) G_{i j(l)}^{T} G_{i j(l)}, \\
& i \in \mathbf{I}[1, r], \quad j \in \mathbf{I}[1, i], \quad l \in \mathbf{I}[1, m] .
\end{aligned}
$$

Moreover, it follows from (37) and (38) that

$$
\begin{gathered}
\left|w_{i j(l)}(t)\right|^{2}=x^{T}(t) G_{i j(l)}^{T} G_{i j(l)} x(t)<\left(\bar{u}_{j(l)}^{2} / \beta_{i}\right) V_{i}(t), \\
t \in\left[t_{i}^{*}, t_{i+1}^{*}\right), \quad i \in \mathbf{I}[1, r], \quad j \in \mathbf{I}[1, i], \quad l \in \mathbf{I}[1, m] .
\end{gathered}
$$

Finally, we will show that, for all initial conditions $x_{0}$ satisfying $V_{0}(0) \leq 1$, the modified sector conditions (10) can be guaranteed and the relations (35) and (36) are valid. Moreover, one can conclude from (36) that the system (6)-(7) is locally exponentially stable.

For $\forall x_{0}$ satisfying $V_{0}(0) \leq 1$, from (30), (33) and (39), we have

$$
\begin{aligned}
\left|w_{11(l)}\left(t_{1}^{*}\right)\right| & <\left[\left(\bar{u}_{1(l)}^{2} / \beta_{1}\right) \mu_{1} e^{\alpha_{0}\left(t_{1}^{*}-t_{0}^{*}\right)} V_{0}(0)\right]^{1 / 2} \\
& \leq \bar{u}_{1(l)}, \quad l \in \mathbf{I}[1, m] .
\end{aligned}
$$

Noting that $\left|w_{11(l)}(t)\right|(l \in \mathbf{I}[1, m])$ are continuous with respect to $t$, from (40), there exists a time interval $\left[t_{1}^{*}, \hat{t}_{1}\right) \subset\left[t_{1}^{*}, t_{2}^{*}\right)$ such that $\left|w_{11(l)}(t)\right| \leq \bar{u}_{1(l)}, t \in\left[t_{1}^{*}, \hat{t}_{1}\right), l \in \mathbf{I}[1, m]$. Then, within $\left[t_{1}^{*}, \hat{t}_{1}\right)$, the sector condition (10) is satisfied for $i=j=1$ and the relation $V_{i}(t) \leq \beta_{i} V_{0}(0)$ is true for $i=1$. Moreover, we have $V_{1}\left(\hat{t}_{1}\right) \leq \beta_{1} V_{0}(0)$, and then one obtains from (39) that $\left|w_{11(l)}\left(\hat{t}_{1}\right)\right|<$ $\bar{u}_{1(l)}, l \in \mathbf{I}[1, m]$. Again, noting that $\left|w_{11(l)}(t)\right|(l \in \mathbf{I}[1, m])$ are continuous, there exists the time interval $\left[\hat{t}_{1}, \hat{t}_{2}\right) \subset\left[t_{1}^{*}, t_{2}^{*}\right)$ such that $\left|w_{11(l)}(t)\right| \leq \bar{u}_{1(l)}, t \in\left[\hat{t}_{1}, \hat{t}_{2}\right), l \in \mathbf{I}[1, m]$. Then, within $\left[\hat{t}_{1}, \hat{t}_{2}\right)$, the sector condition (10) is ensured $(i=j=1)$ and the relation $V_{i}(t) \leq \beta_{i} V_{0}(0)$ holds $(i=1)$. Furthermore, we have $V_{1}\left(\hat{t}_{2}\right) \leq \beta_{1} V_{0}(0)$, and then from (39), we have $\left|w_{11(l)}\left(\hat{t}_{2}\right)\right|<\bar{u}_{1(l)}$, $l \in \mathbf{I}[1, m]$. Repeating the above process, one can conclude that the sector condition (10) is satisfied $(i=j=1)$ and the relation $V_{i}(t) \leq \beta_{i} V_{0}(0)$ denoted in (35) is guaranteed $(i=1)$ within the whole interval $t \in\left[t_{1}^{*}, t_{2}^{*}\right)$. Meanwhile, one has $V_{1}\left(t_{2}^{*}\right) \leq \beta_{1} V_{0}(0)$.

From (33) and (39), it can be seen that

$$
\begin{aligned}
\left|w_{2 j(l)}\left(t_{2}^{*}\right)\right| & <\left[\left(\bar{u}_{j(l)}^{2} / \beta_{2}\right) \mu_{2} \beta_{1} V_{0}(0)\right]^{1 / 2} \\
& \leq \bar{u}_{j(l)}, \quad j \in \mathbf{I}[1,2], \quad l \in \mathbf{I}[1, m] .
\end{aligned}
$$

Using the similar arguments as above, one can prove that the sector condition (10) is satisfied for $i=2, j \in \mathbf{I}[1,2]$ and the relation (35) holds for $i=2$ within the interval $\left[t_{2}^{*}, t_{3}^{*}\right)$. Repeating the above procedure, it is seen that the sector conditions (10) are ensured and the relations (35) and (36) are true. The proof is now complete.

Remark 4: In the proof of Theorem 1, the piecewise L-K functional (11) is utilized to characterize the state evolution of the closed-loop system (6)-(7). Of course, for the asymptotic stability analysis, we can also choose a common Lyapunov functional. Note that the dynamics of the system (6)-(7) has different representations within different time-intervals $\left[t_{\hat{i}}^{*}, t_{\hat{i}+1}^{*}\right)(\hat{i} \in \mathbf{I}[0, r])$. Hence, our proposed functional (11) is more flexible in analyzing the exponential stability of (6)-(7). On the other hand, in the proof of Theorem 1, we have assumed that the system trajectories might diverge with the increasing exponentials $\alpha_{k}$ within the time-intervals $\left[t_{k}^{*}, t_{k+1}^{*}\right)(k \in \mathbf{I}[0, r-1])$ and become convergent with the decreasing exponential $\alpha_{r}$ from the instant $t_{r}^{*}$. In addition, it is worth mentioning that the scalars $\alpha_{k}(k \in \mathbf{I}[0, r-1])$ in Theorem 1 are required to satisfy $\alpha_{0} \geq \alpha_{1} \geq \cdots \geq \alpha_{r-1}$.

To convert the matrix inequalities (12)-(16) in Theorem 1 into LMIs or quasi-LMIs, we set $R_{\hat{i}}=R, S_{\hat{i}}=\delta_{\hat{i}} R\left(\delta_{\hat{i}} \neq 0\right)$ and denote

$$
\left\{\begin{array}{l}
R^{-1} \triangleq X, X P_{\hat{i}} X^{T} \triangleq \bar{P}_{\hat{i}}, X Q_{\hat{i} \hat{j}} X^{T} \triangleq \bar{Q}_{\hat{i} \hat{j}}, \\
X Z_{\hat{i} \hat{j}} X^{T} \triangleq \bar{Z}_{\hat{i} \hat{j}}, X M_{i j} X^{T} \triangleq \bar{M}_{i j}, \varepsilon_{\hat{i}}^{-1} \triangleq \bar{\varepsilon}_{\hat{i}}, \\
K_{i} X^{T} \triangleq \bar{Y}_{i}, G_{i j} X^{T} \triangleq \bar{G}_{i j}, H_{i j}^{-1} \triangleq \bar{H}_{i j} .
\end{array}\right.
$$

Performing some congruence transformations to (12)-(16) in Theorem 1 [2], and using (42), the following local stabilization condition can be readily obtained in terms of LMIs and quasi-LMIs.

Theorem 2: Let the scalars $\alpha_{\hat{i}}>0, \mu_{i}>0$ and $\delta_{\hat{i}} \neq 0$ be given. Assume that there exist $n \times n$ matrices $X, \bar{P}_{\hat{i}}>0, \bar{Q}_{\hat{i} \hat{j}}>0, \bar{Z}_{\hat{i} \hat{j}}>0$, $\bar{M}_{i j}, m \times n$ matrices $Y_{i}, \bar{G}_{i j}, m \times m$ diagonal matrices $\bar{H}_{i j}>0$, and the scalars $\bar{\varepsilon}_{\hat{i}}>0, \hat{i} \in \mathbf{I}[0, r], i, \hat{j} \in \mathbf{I}[1, r], j \in \mathbf{I}[1, i]$, such that for $\forall i, \hat{j} \in \mathbf{I}[1, r], \forall j \in \mathbf{I}[1, i]$, the following LMIs hold:

$$
\begin{aligned}
& {\left[\begin{array}{cc}
\bar{Z}_{i j} & \bar{M}_{i j} \\
\bar{M}_{i j}^{T} & \bar{Z}_{i j}
\end{array}\right]>0} \\
& {\left[\begin{array}{cccc}
\bar{\Omega}_{11}^{0} & \bar{\Omega}_{12}^{0} & \bar{\Omega}_{13}^{0} & \bar{\Omega}_{14}^{0} \\
* & \bar{\Omega}_{22}^{0} & 0 & 0 \\
* & * & -2 \bar{\varepsilon}_{0} I & \delta_{0} \bar{\varepsilon}_{0} I \\
* & * & * & \bar{\Omega}_{44}^{0}
\end{array}\right]<0,} \\
& {\left[\begin{array}{cccccc}
\bar{\Omega}_{11}^{i} & \bar{\Omega}_{12}^{i} & \bar{\Omega}_{13}^{i} & \bar{\Omega}_{14}^{i} & \bar{\Omega}_{15}^{i} & \bar{\Omega}_{16}^{i} \\
* & \bar{\Omega}_{22}^{i} & \bar{\Omega}_{23}^{i} & \bar{\Omega}_{24}^{i} & 0 & \bar{\Omega}_{26}^{i} \\
* & * & \bar{\Omega}_{33}^{i} & 0 & 0 & 0 \\
* & * & * & \bar{\Omega}_{44}^{i} & 0 & \bar{\Omega}_{46}^{i} \\
* & * & * & * & -2 \bar{\varepsilon}_{i} I & \delta_{i} \bar{\varepsilon}_{i} I \\
* & * & * & * & * & \bar{\Omega}_{66}^{i}
\end{array}\right]<0,}
\end{aligned}
$$

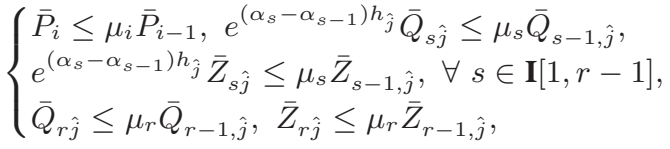

$$
\begin{aligned}
& {\left[\begin{array}{cc}
\bar{u}_{j(l)}^{2} / \beta_{i} & \bar{G}_{i j(l)} \\
\bar{G}_{i j(l)}^{T} & \bar{P}_{i}
\end{array}\right] \geq 0, \forall l \in \mathbf{I}[1, m]}
\end{aligned}
$$

where

$$
\begin{aligned}
& \bar{\Omega}_{11}^{0} \triangleq \operatorname{He}\left(A X^{T}\right)-\alpha_{0} \bar{P}_{0}+\sum_{\hat{j}=1}^{r}\left(\bar{Q}_{0 \hat{j}}-\bar{Z}_{0 \hat{j}}\right), \\
& \bar{\Omega}_{12}^{0} \triangleq\left[\begin{array}{llll}
\bar{Z}_{01} & \bar{Z}_{02} & \cdots & \bar{Z}_{0 r}
\end{array}\right], \bar{\Omega}_{13}^{0} \triangleq \bar{\varepsilon}_{0} I+X F^{T}, \\
& \bar{\Omega}_{22}^{0} \triangleq \operatorname{diag}\left\{-e^{\alpha_{0} h_{1}} \bar{Q}_{01}-\bar{Z}_{01}, \ldots,-e^{\alpha_{0} h_{r}} \bar{Q}_{0 r}-\bar{Z}_{0 r}\right\} \text {, } \\
& \bar{\Omega}_{14}^{0} \triangleq \bar{P}_{0}-X^{T}+\delta_{0} X A^{T}, \bar{\Omega}_{44}^{0} \triangleq \Sigma_{\hat{j}=1}^{r} h_{\hat{j}}^{2} \bar{Z}_{0 \hat{j}}-\delta_{0} \operatorname{He}(X) \text {, } \\
& \bar{\Omega}_{11}^{s} \triangleq \operatorname{He}\left(A X^{T}\right)-\alpha_{s} \bar{P}_{s}+\Sigma_{\hat{j}=1}^{r}\left(\bar{Q}_{s \hat{j}}-\check{Z}_{s \hat{j}}\right), \\
& \bar{\Omega}_{11}^{r} \triangleq \operatorname{He}\left(A X^{T}\right)+\alpha_{r} \bar{P}_{r}+\sum_{\hat{j}=1}^{r}\left(\bar{Q}_{r \hat{j}}-\check{Z}_{r \hat{j}}\right), \\
& \bar{\Omega}_{12}^{i} \triangleq\left[B_{1} Y_{1}+\check{Z}_{i 1}-\check{M}_{i 1} \cdots B_{i} Y_{i}+\check{Z}_{i i}-\check{M}_{i i}\right] \text {, } \\
& \bar{\Omega}_{13}^{s} \triangleq\left[\begin{array}{llllll}
\check{M}_{s 1} & \cdots & \check{M}_{s s} & \check{Z}_{s, s+1} & \cdots & \check{Z}_{s r}
\end{array}\right], \\
& \bar{\Omega}_{14}^{i} \triangleq\left[-B_{1} \bar{H}_{i 1}-\bar{G}_{i 1}^{T} \cdots-B_{i} \bar{H}_{i i}-\bar{G}_{i i}^{T}\right] \text {, } \\
& \bar{\Omega}_{15}^{i} \triangleq \bar{\varepsilon}_{i} I+X F^{T}, \bar{\Omega}_{16}^{i} \triangleq-X^{T}+\delta_{i} X A^{T}+\bar{P}_{i}, \\
& \bar{\Omega}_{22}^{i} \triangleq \operatorname{diag}\left\{\operatorname{He}\left(\check{M}_{i 1}\right)-2 \check{Z}_{i 1}, \ldots, \operatorname{He}\left(\check{M}_{i i}\right)-2 \check{Z}_{i i}\right\} \text {, } \\
& \bar{\Omega}_{23}^{i} \triangleq\left[\operatorname{diag}\left\{\check{Z}_{i 1}-\check{M}_{i 1}, \ldots, \check{Z}_{i i}-\check{M}_{i i}\right\} \quad 0_{i n \times(r-i) n}\right] \text {, } \\
& \bar{\Omega}_{24}^{i} \triangleq \operatorname{diag}\left\{Y_{1}^{T}, \ldots, Y_{i}^{T}\right\}, \bar{\Omega}_{26}^{i} \triangleq \delta_{i}\left[B_{1} Y_{1} \cdots B_{i} Y_{i}\right]^{T} \text {, }
\end{aligned}
$$




$$
\begin{aligned}
& \bar{\Omega}_{33}^{i} \triangleq \operatorname{diag}\left\{-\check{Q}_{i 1}-\check{Z}_{i 1}, \ldots,-\check{Q}_{i r}-\check{Z}_{i r}\right\}, \\
& \bar{\Omega}_{44}^{i} \triangleq-2 \operatorname{diag}\left\{\bar{H}_{i 1}, \ldots, \bar{H}_{i i}\right\}, \bar{\Omega}_{13}^{r} \triangleq\left[\check{M}_{r 1} \cdots \check{M}_{r r}\right], \\
& \bar{\Omega}_{46}^{i} \triangleq-\delta_{i}\left[B_{1} \bar{H}_{i 1} \cdots B_{i} \bar{H}_{i i}\right]^{T}, \\
& \bar{\Omega}_{66}^{i} \triangleq \Sigma_{\hat{j}=1}^{r} h_{\hat{j}}^{2} \bar{Z}_{i \hat{j}}-\delta_{i} \operatorname{He}(X), s \in \mathbf{I}[1, r-1] .
\end{aligned}
$$

Then, for all $x_{0}$ satisfying $V_{0}(0) \leq 1$, the system (1) is exponentially stabilized by the controllers (4) with $K_{i}=Y_{i} X^{-T}, i \in \mathbf{I}[1, r]$.

For the constant-delay case, i.e., $\tau_{i}(t) \equiv h_{i}(i \in \mathbf{I}[1, r], t \geq 0)$, the scalars $t_{i}^{*}(i \in \mathbf{I}[1, r])$ should be substituted with $h_{i}$. Then, one can obtain the following simplified stabilization condition.

Corollary 1: Let the scalars $\alpha_{\hat{i}}>0, \mu_{i}>0$ and $\delta_{\hat{i}} \neq 0$ be given. The conclusion of Theorem 2 is ensured if there exist matrices $X$, $\bar{P}_{\hat{i}}>0, \bar{Q}_{\hat{i} \hat{j}}>0, \bar{Z}_{\hat{i} \hat{j}}>0, Y_{i}, \bar{G}_{i j}$, diagonal matrices $\bar{H}_{i j}>0$, and scalars $\bar{\varepsilon}_{\hat{i}}>0, \hat{i} \in \mathbf{I}[0, r], i, \hat{j} \in \mathbf{I}[1, r], j \in \mathbf{I}[1, i]$, such that for $\forall i, \hat{j} \in \mathbf{I}[1, r], \forall j \in \mathbf{I}[1, i]$, the LMIs (44), (46), (47) and

$$
\left[\begin{array}{ccccc}
\check{\Omega}_{11}^{i} & \check{\Omega}_{12}^{i} & \check{\Omega}_{13}^{i} & \check{\Omega}_{14}^{i} & \check{\Omega}_{15}^{i} \\
* & \check{\Omega}_{22}^{i} & \check{\Omega}_{23}^{i} & 0 & \check{\Omega}_{25}^{i} \\
* & * & \check{\Omega}_{33}^{i} & 0 & \check{\Omega}_{35}^{i} \\
* & * & * & -2 \bar{\varepsilon}_{i} I & \delta_{i} \bar{\varepsilon}_{i} I \\
* & * & * & * & \check{\Omega}_{55}^{i}
\end{array}\right]<0
$$

are feasible, where

$$
\begin{aligned}
& \check{\Omega}_{11}^{s} \triangleq \operatorname{He}\left(A X^{T}\right)-\alpha_{s} \bar{P}_{s}+\Sigma_{\hat{j}=1}^{r}\left(\bar{Q}_{s \hat{j}}-\check{Z}_{s \hat{j}}\right), \\
& \check{\Omega}_{11}^{r} \triangleq \operatorname{He}\left(A X^{T}\right)+\alpha_{r} \bar{P}_{i}+\sum_{\hat{j}=1}^{r}\left(\bar{Q}_{r \hat{j}}-\check{Z}_{r \hat{j}}\right), \\
& \check{\Omega}_{12}^{s} \triangleq\left[\begin{array}{llllll}
B_{1} Y_{1}+\check{Z}_{s 1} & \cdots & B_{s} Y_{s}+\check{Z}_{s s} & \check{Z}_{s, s+1} & \cdots & \check{Z}_{s r}
\end{array}\right] \text {, } \\
& \check{\Omega}_{12}^{r} \triangleq\left[B_{1} Y_{1}+\check{Z}_{r 1} \cdots B_{r} Y_{r}+\check{Z}_{r r}\right] \text {, } \\
& \check{\Omega}_{13}^{i} \triangleq\left[-B_{1} \bar{H}_{i 1}-\bar{G}_{i 1}^{T} \cdots-B_{i} \bar{H}_{i i}-\bar{G}_{i i}^{T}\right] \text {, } \\
& \check{\Omega}_{14}^{i} \triangleq \bar{\varepsilon}_{i} I+X F^{T}, \check{\Omega}_{15}^{i} \triangleq \bar{P}_{i}-X^{T}+\delta_{i} X A^{T} \text {, } \\
& \check{\Omega}_{22}^{i} \triangleq \operatorname{diag}\left\{-\check{Q}_{i 1}-\check{Z}_{i 1}, \ldots,-\check{Q}_{i r}-\check{Z}_{i r}\right\} \text {, } \\
& \check{\Omega}_{23}^{i} \triangleq\left[\operatorname{diag}\left\{Y_{1}, \ldots, Y_{i}\right\} \quad 0_{i m \times(r-i) n}\right]^{T} \text {, } \\
& \check{\Omega}_{25}^{i} \triangleq\left[\begin{array}{llll}
\delta_{i} B_{1} Y_{1} & \cdots & \delta_{i} B_{i} Y_{i} & 0_{i n \times(r-i) n}
\end{array}\right]^{T}, \\
& \check{\Omega}_{33}^{i} \triangleq \operatorname{diag}\left\{-2 \bar{H}_{i 1}, \ldots,-2 \bar{H}_{i i}\right\} \text {, } \\
& \check{\Omega}_{35}^{i} \triangleq\left[\begin{array}{lll}
-\delta_{i} B_{1} \bar{H}_{i 1} & \cdots & -\delta_{i} B_{i} \bar{H}_{i i}
\end{array}\right]^{T}, \\
& \check{\Omega}_{55}^{i} \triangleq \Sigma_{\hat{j}=1}^{r} h_{\hat{j}}^{2} \bar{Z}_{i \hat{j}}-\delta_{i} \operatorname{He}(X), s \in \mathbf{I}[1, r-1] .
\end{aligned}
$$

If the system (1) contains only a single time-varying input delay $\tau_{1}(t) \in\left[0, h_{1}\right]$, then Theorem 2 can be simplified as follows.

Corollary 2: Let the scalars $\alpha_{\hat{i}}>0, \mu_{1}>0$ and $\delta_{\hat{i}} \neq 0$ be given. The conclusion of Theorem 2 is guaranteed if there exist matrices $X, \bar{P}_{\hat{i}}>0, \bar{Q}_{\hat{i} 1}>0, \bar{Z}_{\hat{i} 1}>0, \bar{M}_{11}, Y_{1}, \bar{G}_{11}$, diagonal matrices $\bar{H}_{11}>0$, and scalars $\bar{\varepsilon}_{\hat{i}}>0, \hat{i}=0,1$, such that the LMIs

$$
\begin{aligned}
& {\left[\begin{array}{cccc}
\hat{\Omega}_{11}^{0} & \bar{Z}_{01} & \hat{\Omega}_{13}^{0} & \hat{\Omega}_{14}^{0} \\
* & \hat{\Omega}_{22}^{0} & 0 & 0 \\
* & * & -2 \bar{\varepsilon}_{0} I & \delta_{0} \bar{\varepsilon}_{0} I \\
* & * & * & \hat{\Omega}_{44}^{0}
\end{array}\right]<0,} \\
& {\left[\begin{array}{cccccc}
\hat{\Omega}_{11}^{1} & \hat{\Omega}_{12}^{1} & \hat{\Omega}_{13}^{1} & \hat{\Omega}_{14}^{1} & \hat{\Omega}_{15}^{1} & \hat{\Omega}_{16}^{1} \\
* & \hat{\Omega}_{22}^{1} & \hat{\Omega}_{23}^{1} & Y_{1}^{T} & 0 & \hat{\Omega}_{26}^{1} \\
* & * & \hat{\Omega}_{33}^{1} & 0 & 0 & 0 \\
* & * & * & -2 \bar{H}_{11} & 0 & \hat{\Omega}_{46}^{1} \\
* & * & * & * & -2 \bar{\varepsilon}_{1} I & \delta_{1} \bar{\varepsilon}_{1} I \\
* & * & * & * & * & \hat{\Omega}_{66}^{1}
\end{array}\right]<0,} \\
& \bar{P}_{1} \leq \mu_{1} \bar{P}_{0}, \bar{Q}_{11} \leq \mu_{1} \bar{Q}_{01}, \bar{Z}_{11} \leq \mu_{1} \bar{Z}_{01}, \\
& {\left[\begin{array}{cc}
\bar{Z}_{11} & \bar{M}_{11} \\
\bar{M}_{11}^{T} & \bar{Z}_{11}
\end{array}\right]>0,\left[\begin{array}{cc}
\bar{u}_{1(l)}^{2} / \hat{\beta} & \bar{G}_{11(l)} \\
\bar{G}_{11(l)}^{T} & \bar{P}_{1}
\end{array}\right] \geq 0, \forall l \in \mathbf{I}[1, m]}
\end{aligned}
$$

are satisfied, where

$$
\hat{\Omega}_{11}^{0} \triangleq-\alpha_{0} \bar{P}_{0}+\operatorname{He}\left(A X^{T}\right)+\bar{Q}_{01}-\bar{Z}_{01},
$$

$$
\begin{aligned}
& \hat{\Omega}_{13}^{0} \triangleq \bar{\varepsilon}_{0} I+X F^{T}, \hat{\Omega}_{14}^{0} \triangleq-X^{T}+\delta_{0} X A^{T}+\bar{P}_{0}, \\
& \hat{\Omega}_{22}^{0} \triangleq-e^{\alpha_{0} h_{1}} \bar{Q}_{01}-\bar{Z}_{01}, \hat{\Omega}_{44}^{0} \triangleq h_{1}^{2} \bar{Z}_{01}-\delta_{0} \operatorname{He}(X), \\
& \hat{\Omega}_{11}^{1} \triangleq \operatorname{He}\left(A X^{T}\right)+\alpha_{1} \bar{P}_{1}+\bar{Q}_{11}-e^{-\alpha_{1} h_{1}} \bar{Z}_{11}, \\
& \hat{\Omega}_{12}^{1} \triangleq B_{1} Y_{1}+e^{-\alpha_{1} h_{1}}\left(\bar{Z}_{11}-\bar{M}_{11}\right), \hat{\Omega}_{13}^{1} \triangleq e^{-\alpha_{1} h_{1}} \bar{M}_{11}, \\
& \hat{\Omega}_{14}^{1} \triangleq-B_{1} \bar{H}_{11}-\bar{G}_{11}^{T}, \hat{\Omega}_{15}^{1} \triangleq \bar{\varepsilon}_{1} I+X F^{T}, \\
& \hat{\Omega}_{16}^{1} \triangleq-X^{T}+\delta_{1} X A^{T}+\bar{P}_{1}, \hat{\Omega}_{26}^{1} \triangleq \delta_{1} Y_{1}^{T} B_{1}^{T}, \\
& \hat{\Omega}_{22}^{1} \triangleq e^{-\alpha_{1} h_{1}}\left[\operatorname{He}\left(\bar{M}_{11}\right)-2 \bar{Z}_{11}\right], \hat{\Omega}_{46}^{1} \triangleq-\delta_{1} \bar{H}_{11}^{T} B_{1}^{T}, \\
& \hat{\Omega}_{23}^{1} \triangleq e^{-\alpha_{1} h_{1}}\left(\bar{Z}_{11}-\bar{M}_{11}\right), \hat{\Omega}_{33}^{1} \triangleq-e^{-\alpha_{1} h}\left(\bar{Q}_{11}+\bar{Z}_{11}\right), \\
& \hat{\Omega}_{66}^{1} \triangleq-\delta_{1} \operatorname{He}(X)+h_{1}^{2} \bar{Z}_{11}, \hat{\beta} \triangleq e^{\alpha_{0}\left(t_{1}^{*}-t_{0}^{*}\right)} \mu_{1} .
\end{aligned}
$$

Remark 5: In [14], the local stabilization problem has been studied for linear systems with a single input delay under actuator saturations. Unlike [14], the piecewise L-K functional and the modified sector condition are utilized in this paper to reduce the conservatism. Using the piecewise L-K functional (11) (the case $r=1$ ), one can perform the more flexible analysis within the first time-interval $\left[t_{0}^{*}, t_{1}^{*}\right)$, since we utilize the functional $V_{0}(t)$ instead of $V_{1}(t)$ within the interval $\left[t_{0}^{*}, t_{1}^{*}\right)$ [14]. Using the modified sector condition (10) (the case $r=$ 1 ), the design variables $\bar{G}_{11}$ and $Y_{1}$ can be introduced in two different positions of LMI (50), which makes that it is more flexible to select the variables $\bar{G}_{11}$ and $Y_{1}$ to ensure the feasibility of LMI (50).

Remark 6: For the case that $t_{i}^{*}(i \in \mathbf{I}[1, r])$ are not exactly known, using the fact $t_{i}^{*} \leq h_{i}$, the scalars $\beta_{i}(i \in \mathbf{I}[1, r-1])$ in Theorem $1-2$ can be revised as $\beta_{i}=e^{\alpha_{0} h_{1}+\alpha_{1} h_{2}+\cdots+\alpha_{i} h_{i+1}} \mu_{1} \cdots \mu_{i}$ or $\beta_{i}=$ $e^{\alpha_{0} h_{i+1}} \mu_{1} \cdots \mu_{i}$. Meanwhile, in Corollary $2, \hat{\beta}$ can be set as $\hat{\beta}=$ $e^{\alpha_{0} h_{1}} \mu_{1}$. If the relation (5) in Assumption 1 is removed, we have to consider all possibilities concerning the scalars $t_{0}^{*}, t_{1}^{*}, t_{2}^{*}, \cdots, t_{r}^{*}$. For example, for the case $r=2$, the relation $t_{0}^{*}<t_{2}^{*}<t_{1}^{*} \leq h_{1}$ should be additionally addressed and some conditions should be added.

Remark 7: The main objective of this paper is to establish the local stabilization conditions for multiple input-delay systems under actuator saturations by developing some more effective techniques. Our proposed results are based on the simple state feedback controllers (4). Here, it is worth mentioning that it is not difficult to establish the corresponding results by using dynamic output feedback controllers. Moreover, by incorporating the time delay information into the feedback controllers [5], [29], [35], we can also address the predictor-based control design problem, which is our further work.

Next, we will be concerned with the estimate of the DOA. As in [14], [22], we will determine an ellipsoid that is contained in the DOA. Here, we assume that the ellipsoid has the following form:

$$
\mathscr{E} \triangleq\left\{x_{0} \in \mathbb{R}^{n}: x_{0}^{T} \mathcal{P} x_{0} \leq 1, \mathcal{P}>0\right\} .
$$

Noting that $x(s)=x_{0}, s \in[-h, 0]$, it follows from (11) and (42) that the admissible initial conditions $x_{0}$ satisfy

$$
V_{0}(0)=x_{0}^{T} X^{-1} \check{\mathcal{P}} X^{-T} x_{0} \triangleq x_{0}^{T} \mathcal{P} x_{0} \leq 1
$$

where $\left.\check{\mathcal{P}} \triangleq \bar{P}_{0}+\Sigma_{\hat{j}=1}^{r}\left(e^{\alpha_{0} h_{\hat{j}}}-1\right) / \alpha_{0}\right) \bar{Q}_{0 \hat{j}}$.

Let us introduce the following matrix inequality:

$$
X^{-1} \check{\mathcal{P}} X^{-T} \leq p I
$$

where $p>0$ is a scalar. Similar to the arguments in [14], [20], the matrix inequality (55) can be guaranteed by the following LMI:

$$
\left[\begin{array}{cc}
p I & I \\
I & X+X^{T}-\check{\mathcal{P}}
\end{array}\right] \geq 0 .
$$

Then, the optimization problems about the initial condition set $\mathscr{E}$ in Theorem 2 and Corollaries 1-2 can be, respectively, formulated as

$$
\text { Prob.1. } \min _{X, \bar{P}_{\hat{i}}>0, \bar{Q}_{\hat{i} \hat{j}}>0, \bar{Z}_{\hat{i} \hat{j}}>0, \bar{M}_{i j}, Y_{i}, \bar{G}_{i j}, \bar{H}_{i j}>0, \bar{\varepsilon}_{\hat{i}}>0, p>0} p,
$$




$$
\begin{aligned}
& \text { s.t., LMIs (43) - (47) and (56) hold, } \\
& \text { Prob.2. }{ }_{X, \bar{P}_{\hat{i}}>0, \bar{Q}_{\hat{i} \hat{j}}>0, \bar{Z}_{\hat{i} \hat{j}}>0, Y_{i}, \bar{G}_{i j}, \bar{H}_{i j}>0, \bar{\varepsilon}_{\hat{i}}>0, p>0} p \text {, s.t., } \\
& \text { LMIs (44), (46) - (48) and (56) hold, } \\
& \begin{array}{c}
\text { Prob.3. } \\
\min _{X, \bar{P}_{\hat{i}}>0, \bar{Q}_{\hat{i} 1}>0, \bar{Z}_{\hat{i} 1}>0, \bar{M}_{11}, Y_{1}, \bar{G}_{11}, \bar{H}_{11}>0, \bar{\varepsilon}_{\hat{\imath}}>0, p>0} p, \\
\text { s.t., LMIs (49) - (52) and (56) }(\mathrm{r}=1) \text { hold. }
\end{array}
\end{aligned}
$$

\section{NUMERICAL EXAMPLES}

Example 1: [24] Consider the synchronization control for the following dynamical network subject to actuator saturations:

$$
\left\{\begin{array}{l}
\dot{x}_{i}(t)=\tilde{f}\left(x_{i}(t)\right)+\sum_{j=1}^{N} c_{i j} \Gamma x_{j}(t)+\operatorname{sat}\left(u_{i}(t)\right) \\
x_{i}(\theta)=x_{i 0}, \quad \theta \in[-h, 0], \quad i \in \mathbf{I}[1, N]
\end{array}\right.
$$

where $x_{i}(t) \in \mathbb{R}^{n}, u_{i}(t) \in \mathbb{R}^{n}, x_{i 0} \in \mathbb{R}^{n}$, are, respectively, the state vector, the control input and the initial condition of the $i$-th node; $\Gamma$ is the inner-coupling matrix, and $C=\left(c_{i j}\right)_{N \times N}$ is the symmetric outer-coupling matrix with $c_{i j} \geq 0(i \neq j)$ and $c_{i i}=$ $-\sum_{j=1, j \neq i}^{N} c_{i j}(i \in \mathbf{I}[1, N])$; $\operatorname{sat}\left(u_{i}\right)$ has the same meaning as in (1); $f(x)$ is continuous with $\tilde{f}(0)=0$ and satisfies

$$
\begin{aligned}
& {\left[\tilde{f}(x)-\tilde{f}(y)-F_{1}(x-y)\right]^{T}} \\
& \quad \times\left[\tilde{f}(x)-\tilde{f}(y)-F_{2}(x-y)\right] \leq 0 .
\end{aligned}
$$

The unforced isolate node is given as follows:

$$
\dot{s}(t)=\tilde{f}(s(t)), s(\theta)=s_{0}, \theta \in[-h, 0] .
$$

Let us denote in this section that $e_{i}(t) \triangleq x_{i}(t)-s(t)$ and

$$
\begin{aligned}
& e(t) \triangleq\left[\begin{array}{llll}
e_{1}^{T}(t) & e_{2}^{T}(t) & \cdots & \left.e_{N}^{T}(t)\right)
\end{array}\right]^{T}, \\
& f\left(e_{i}(t)\right) \triangleq \tilde{f}\left(x_{i}(t)\right)-\tilde{f}(s(t))-F_{1} e_{i}(t), \\
& \left.f(e(t)) \triangleq\left[f^{T}\left(e_{1}(t)\right) \quad f^{T}\left(e_{2}(t)\right) \cdots f^{T}\left(e_{N}(t)\right)\right)\right]^{T}, \\
& e_{i 0} \triangleq x_{i 0}-s_{0}, e_{0} \triangleq\left[\begin{array}{llll}
e_{10}^{T} & e_{20}^{T} & \cdots & e_{N 0}^{T}
\end{array}\right]^{T}, \\
& A \triangleq I_{N \times N} \otimes F_{1}+C \otimes \Gamma, B_{i} \triangleq \epsilon_{i} \otimes I_{n \times n}
\end{aligned}
$$

where $\epsilon_{i} \in \mathbb{R}^{1 \times N}$ is a row vector whose $i$-th element is 1 and others are zero. Then, we have the following error dynamics:

$$
\left\{\begin{array}{l}
\dot{e}(t)=A e(t)+f(e(t))+\sum_{i=1}^{N} B_{i} \operatorname{sat}\left(u_{i}(t)\right) \\
e(t)=e_{0}, \theta \in[-\tau, 0]
\end{array}\right.
$$

where $f(e)$ satisfies (3) with $F \triangleq I_{N \times N} \otimes\left(F_{2}-F_{1}\right)$.

It is assumed that the error states $e_{i}(t)(i \in \mathbf{I}[1, N])$ can be sampled at the instants $0=s_{0}^{i}<s_{1}^{i}<\cdots<s_{k}^{i}<\cdots$. Here, we are interested in designing the following sampled-data controllers:

$$
\begin{aligned}
& u_{i}(t)=K_{i} e_{i}\left(t_{k}^{i}-\rho_{k}^{i}\right)\left(t_{k}^{i}=s_{k}^{i}+\rho_{k}^{i}\right), \\
& t_{k}^{i} \leq t<t_{k+1}^{i}, k=0,1,2, \ldots, i \in \mathbf{I}[1, N]
\end{aligned}
$$

where $\rho_{k}^{i}(i \in \mathbf{I}[1, N])$ denote the transmission delays with $0 \leq \rho_{k}^{i} \leq$ $\rho_{i}$. Let us introduce the following notation:

$$
\tau_{i}(t) \triangleq t-t_{k}^{i}+\rho_{k}^{i}, t_{k}^{i} \leq t<t_{k+1}^{i} .
$$

Then, the sampled-data controllers (61) can be rewritten as

$$
u_{i}(t)=\epsilon_{i} \otimes K_{i} e\left(t-\tau_{i}(t)\right), i \in \mathbf{I}[1, N]
$$

where $0 \leq \tau_{i}(t) \leq t_{k+1}^{i}-t_{k}^{i}+\rho_{i} \leq h_{i}(k=0,1,2, \ldots)$.

For this example, we set $N=3, \Gamma=0.1 I$ and

$$
\begin{aligned}
\tilde{f}\left(x_{i}(t)\right) & =\left[\begin{array}{c}
-0.5 x_{i 1}(t)+\tanh \left(0.2 x_{i 1}(t)\right)+0.2 x_{i 2}(t) \\
0.3 x_{i 2}(t)-\tanh \left(0.1 x_{i 2}(t)\right)
\end{array}\right], \\
\bar{u}_{i(l)} & =10, \quad i=1,2,3, \quad l=1,2, c_{12}=0, \\
c_{11} & =c_{22}=-c_{13}=-c_{23}=-1, c_{33}=-2,
\end{aligned}
$$

$$
\rho_{k}^{1}=0.1, \rho_{k}^{2}=0.2, \rho_{k}^{3}=0.3, t_{k+1}^{i}-t_{k}^{i} \leq 0.5 .
$$

It is seen that $\tau_{i}(t)(i=1,2,3)$ satisfy Assumption 1 with $t_{1}^{*}=$ $0.1, t_{2}^{*}=0.2, t_{3}^{*}=0.3, h_{1}=0.6, h_{2}=0.7$ and $h_{3}=0.8$. Also, it is obvious that $\tilde{f}(x)$ satisfies (58) with

$$
F_{1}=\left[\begin{array}{cc}
-0.5 & 0.2 \\
0 & 0.2
\end{array}\right], F_{2}=\left[\begin{array}{cc}
-0.3 & 0.2 \\
0 & 0.3
\end{array}\right]
$$

Replacing $Y_{i}$ in (45) with $\epsilon_{i} \otimes Y_{i}\left(Y_{i} \in \mathbb{R}^{2 \times 2}\right)$ and solving Prob.1 with $\alpha_{0}=0.67, \alpha_{1}=0.61, \alpha_{2}=0.31, \alpha_{3}=0.1, \delta_{0}=\delta_{i}=\mu_{i}=$ 1 , and $X=\operatorname{diag}\left\{X_{1}, X_{2}, X_{3}\right\}\left(X_{1}, X_{2}, X_{3} \in \mathbb{R}^{2 \times 2}\right)$, we have

$$
\begin{aligned}
K_{1} & =\left[\begin{array}{ll}
-0.1411 & -0.1849 \\
-0.0187 & -0.5429
\end{array}\right], K_{2}=\left[\begin{array}{ccc}
-0.5458 & -0.2032 \\
-0.0097 & -0.8636
\end{array}\right], \\
K_{3} & =\left[\begin{array}{lllll}
-0.5537 & -0.1824 \\
-0.0020 & -0.8550
\end{array}\right], p=34.0402, \mathcal{P}=10^{-3} \\
\times & {\left[\begin{array}{cccccc}
0.3294 & 0.0015 & -0.0003 & -0.0000 & -0.0003 & -0.0001 \\
0.0015 & 0.8630 & 0.0000 & -0.0000 & -0.0000 & -0.0000 \\
-0.0003 & 0.0000 & 0.3293 & -0.0001 & -0.0002 & 0.0000 \\
-0.0000 & -0.0000 & -0.0001 & 0.8630 & -0.0001 & -0.0000 \\
-0.0003 & -0.0000 & -0.0002 & -0.0001 & 0.3177 & 0.0030 \\
-0.0001 & -0.0000 & 0.0000 & -0.0000 & 0.0030 & 0.8516
\end{array}\right] . }
\end{aligned}
$$

In the simulation, the initial conditions are chosen as $x_{10}=$ [15 23], $x_{20}=\left[\begin{array}{ll}12 & 20\end{array}\right], x_{30}=\left[\begin{array}{ll}5 & 10\end{array}\right]$ and $s_{0}=\left[\begin{array}{ll}1 & 0\end{array}\right]$ such that $e_{0}=\left[\begin{array}{llllll}14 & 23 & 11 & 20 & 4 & 10\end{array}\right]$ satisfies $e_{0}^{T} \mathcal{P} e_{0} \leq 1$. It is seen from Figs. 1-2 that the synchronization can be successfully achieved. From Fig. 3, it is seen that the signals $w_{i j(l)}(t)(i \in \mathbf{I}[1,3], j \in$ $\mathbf{I}[1, i], l \in \mathbf{I}[1,2])$ satisfies the constrain conditions (9).

In [24], the following sampled-data controllers are employed:

$$
u_{i}(t)=K e_{i}\left(t-t_{k}\right), t_{k} \leq t<t_{k+1}, i \in \mathbf{I}[1, N]
$$

where $t_{k}\left(t_{0}=0, k=0,1,2, \ldots\right)$ are sampling instants. In this case, the closed-loop error dynamics has the following form:

$$
\left\{\begin{array}{l}
\dot{e}(t)=A e(t)+f(e(t))+\operatorname{sat}(\hat{K} e(t-\tau(t))), \\
e(t)=e_{0}, \theta \in[-\tau, 0]
\end{array}\right.
$$

where $\hat{K} \triangleq I_{N \times N} \otimes K$ and $\tau(t) \triangleq t-t_{k}\left(t_{k} \leq t<t_{k+1}\right)$.

Assume that the sampling is variable and satisfies the relation $\left[t_{k}, t_{k+1}\right) \subset\left[\begin{array}{ll}0.3 & 0.5\end{array}\right)$. By using the optimization problem in [24] (Case I) with $\alpha=0$ and $\kappa=6$, one obtains that

$$
\hat{P}=I_{3 \times 3} \otimes 10^{-3}\left[\begin{array}{cc}
0.7802 & 0 \\
0 & 0.9026
\end{array}\right] .
$$

By solving the optimization problem Prob.3 of this paper with $\alpha_{0}=$ $\mu_{1}=1, \alpha_{1}=t_{1}^{*}=0, \delta_{0}=0.35$ and $\delta_{1}=0.47$, we have $\mathcal{P}=10^{-3}$

$$
\times\left[\begin{array}{cccccc}
0.1439 & -0.0000 & -0.0036 & -0.0001 & -0.0014 & -0.0000 \\
-0.0000 & 0.4547 & -0.0001 & -0.0000 & -0.0001 & 0.0000 \\
-0.0036 & -0.0001 & 0.1439 & -0.0000 & -0.0014 & -0.0000 \\
-0.0001 & -0.0000 & -0.0000 & 0.4547 & -0.0001 & 0.0000 \\
-0.0014 & -0.0001 & -0.0014 & -0.0001 & 0.1380 & 0.0003 \\
-0.0000 & 0.0000 & -0.0000 & 0.0000 & 0.0003 & 0.4547
\end{array}\right] .
$$

Noting that $\hat{P}-\mathcal{P}>0$, it is obvious that our result can provide a large estimate of the DOA than that in [24]. The possible reason for conservatism of the results in [24] is that the matrix variables are set as diagonal to apply the proposed decoupling technique. By utilizing the time-dependent Lyapunov functionals [5], [24], we can obtain more effective results under the sampled-data control scheme.

Example 2: [14] Consider the system (1) with a single delay $\tau_{1}(t) \in\left[0, h_{1}\right]$, where other parameters are given as follows:

$$
\begin{aligned}
& A=\left[\begin{array}{cc}
1.1 & -0.6 \\
0.5 & -1
\end{array}\right], f(x)=\left[\begin{array}{l}
0 \\
0
\end{array}\right], B_{1}=\left[\begin{array}{l}
1 \\
1
\end{array}\right], \\
& \bar{u}_{1(1)}=5, t_{1}^{*} \leq h_{1}\left(t_{1}^{*} \text { is unknown }\right) .
\end{aligned}
$$


For this example, let us first consider the case $h_{1}=0.73$. By solving the optimization problem in [14] with $\sigma=0.001, \alpha=0$, $\beta=1.0, \varepsilon=2.0$ and $\delta=1.85$, it follows that $\varrho=0.7102$ and

$$
\begin{aligned}
K & =\left[\begin{array}{ll}
-1.7432 & 0.5375
\end{array}\right]\left(h_{1}=0.73\right) \\
P & =\left[\begin{array}{cc}
1.8106 & -0.5583 \\
-0.5583 & 0.1722
\end{array}\right] \quad\left(h_{1}=0.73\right) .
\end{aligned}
$$

Letting $\alpha_{0}=2.8, \alpha_{1}=0, \delta_{0}=1.3, \delta_{1}=1.83$ and $\mu_{1}=1.23$, and solving Prob. 3 of this paper, we have $p=1.0520$ and

$$
\begin{aligned}
& K_{1}=\left[\begin{array}{ll}
-1.8525 & 0.5712
\end{array}\right]\left(h_{1}=0.73\right), \\
& \mathcal{P}=\left[\begin{array}{cc}
0.8252 & -0.2545 \\
-0.2545 & 0.0785
\end{array}\right] \quad\left(h_{1}=0.73\right) .
\end{aligned}
$$

Similarly, for the case $h_{1}=0.365$, by solving the optimization problem in [14] with $\sigma=0.001, \alpha=0, \beta=1.0, \varepsilon=0.8$ and $\delta=1.28$, one obtains $\varrho=1.9767, K=\left[\begin{array}{lll}-1.5149 & 0.4671\end{array}\right]$ and

$$
P=\left[\begin{array}{cc}
0.2337 & -0.0721 \\
-0.0721 & 0.0222
\end{array}\right] \quad\left(h_{1}=0.365\right) \text {. }
$$

By using Prob. 3 of this paper with $\alpha_{0}=2.17, \alpha_{1}=0, \delta_{0}=\delta_{1}=0.4$ and $\mu_{1}=1$, we have $p=2.3514, K_{1}=\left[\begin{array}{ll}-2.4227 & 0.7471\end{array}\right]$ and

$$
\mathcal{P}=\left[\begin{array}{cc}
0.1652 & -0.0509 \\
-0.0509 & 0.0157
\end{array}\right] \quad\left(h_{1}=0.365\right) \text {. }
$$

Note that the constraints $\left|K_{i} x(t)\right| \leq \bar{u}_{i}\left(i=1,2, \cdots n_{u}\right)$ are imposed and the saturations are avoided in [14]. In [5], the generalized sector condition is employed to address the same problem. However, for this example, it is declared in [5] that the further application of generalized sector condition cannot enlarge the estimate of the DOA.

Remark 8: In this paper, some scalars are introduced in our main results. In solving Prob.1, the scalars $\alpha_{\hat{i}}, \mu_{i}$ and $\delta_{\hat{i}}(i \in \mathbf{I}[1, r], \hat{i} \in$ $\mathbf{I}[0, r])$ can be determined according to the following steps.

Step 1. For a given $\delta_{0} \triangleq \delta_{0}^{0}$, selecting $\alpha_{0}$ such that LMI (44) is feasible and reducing $\alpha_{0}$ iteratively until LMI (44) is infeasible, we have a smaller $\alpha_{0}$ such that LMI (44) is feasible. Performing the similar search at each iteration $\delta_{0}^{k+1}=\delta_{0}^{k}+d$ ( $d$ is a scalar), one can obtain $\delta_{0}$ within the neighbourhood of $\delta_{0}^{0}$ such that $\alpha_{0}$ is minimum and (44) is feasible. Similarly, for each $i \in \mathbf{I}[1, r-1]$, search $\delta_{i}$ such that $\alpha_{i}$ is minimum and LMIs (43) and (45) are feasible. Also, select $\alpha_{r}$ and $\delta_{r}$ such that LMIs (43) and (45) are feasible for $i=r$.

Step 2. Using $\delta_{\hat{i}}(\hat{i} \in \mathbf{I}[0, r])$ obtained in Step 1 and setting $\mu_{i}=1$ $(i \in \mathbf{I}[1, r])$, increase the scalars $\alpha_{k}(k \in \mathbf{I}[0, r-1])$ obtained in Step 1 iteratively such that Prob. 1 is feasible and the scalar $p$ is maximum.

Step 3. Using $\alpha_{\hat{i}}(\hat{i} \in \mathbf{I}[0, r])$ obtained in Step 2 and setting $\mu_{i}=$ $1(i \in \mathbf{I}[1, r])$, determine $\delta_{\hat{i}}(\hat{i} \in \mathbf{I}[0, r])$ along the similar search procedure as in Step 1 such that $p$ is maximum and Prob.1 is feasible.

Step 4. Using $\alpha_{\hat{i}}$ and $\delta_{\hat{i}}(\hat{i} \in \mathbf{I}[0, r])$ updated in Steps 2-3, search $\mu_{i}(i \in \mathbf{I}[1, r])$ such that $p$ is maximum and Prob. 1 is feasible.

If Step 2 fails, one can reduce the scalar $\alpha_{r}$. To further reduce the conservatism, we can repeat Steps 2-4. The parameters in Prob.2 and Prob. 3 can be determined by using the similar procedure. The procedure proposed above can be used to find feasible solutions and to solve the actual optimization problem in a more efficient way with less conservatism. However, it should be pointed out that the search of these parameters can be extremely complex if $r$ is too large.

\section{Conclusions}

Based on the modified sector conditions, the piecewise L-K functional and the rigorous mathematical deduction, an LMI-based local stabilization condition has been established for a class of multiple input-delay systems with actuator saturations. Two special cases with either constant delays or a single delay have also been considered.

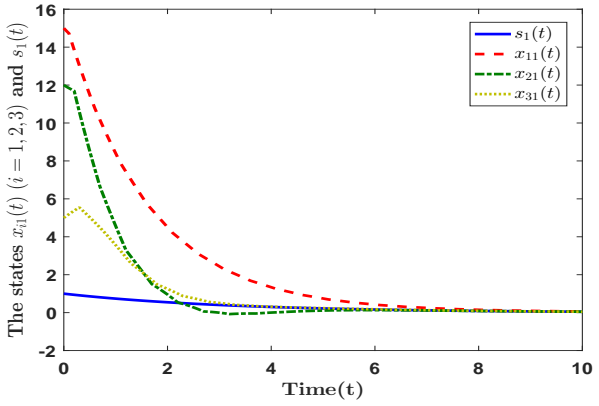

Fig. 1. State evolutions of dynamical network and isolate node.

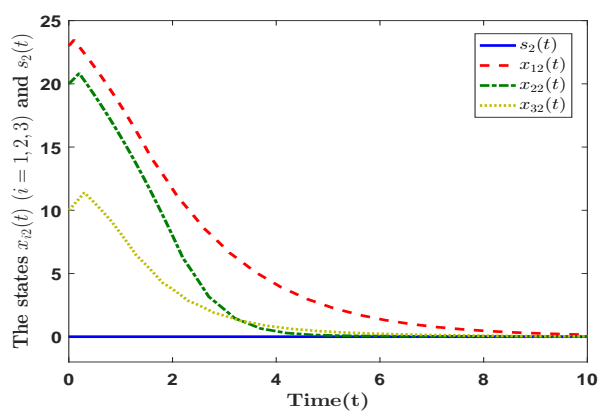

Fig. 2. State evolutions of dynamical network and isolate node.

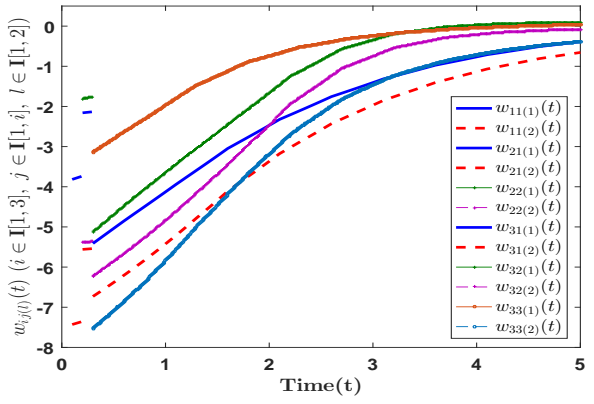

Fig. 3. The evolutions of $w_{i j(l)}(t)(i \in \mathbf{I}[1,3], j \in \mathbf{I}[1, i], l \in \mathbf{I}[1,2])$.

The optimization problems have been formulated to maximize the estimate of the DOA. Finally, two simulation examples have been presented to illustrate the effectiveness and values of our results.

The main results in this paper are based on the slowly-varying delays under which Assumption 1 can be satisfied. In reality, the time delays may be fast varying. In this case, there may exist multiple dynamics within every interval $\left[t_{k}^{*}, t_{k+1}^{*}\right)(k \in \mathbf{I}[0, r-1])$. Hence, our main results cannot be directly applicable. The further analysis should be performed and some additional conditions should be added.

For control systems with multiple inputs, the polytopic models may be more effective in handling the saturations. Under the constraints (9), using the polytopic model approach [8], [22], the saturated inputs $\operatorname{sat}\left(u_{i}\left(t-\tau_{i}(t)\right)\right)(i \in \mathbf{I}[1, r])$ can be written as the combinations of the delayed states and the current state. By further incorporating the analysis approach in this paper, the local stabilization conditions based on the polytopic models can be readily established. However, it should be pointed out that more LMIs will be introduced in the obtained results, which will complicate the computation greatly.

In addition, it is worth pointing out that the technique handling the 
time delays in this paper is based on the traditional Jensen integral inequality. Therefore, our proposed results are conservative to some extent. As the further research, we would like to establish the less conservative stabilization conditions by incorporating the augmented L-K functionals and the new developed inequalities [16], [21], [31].

\section{REFERENCES}

[1] Y. Chen, S. Fei, and Y. Li, "Robust stabilization for uncertain saturated time-delay systems: a distributed-delay-dependent polytopic approach", IEEE Trans. Autom. Control, vol. 62, no. 7, pp. 3455-3460, Jul. 2017.

[2] Y. Chen, Z. Wang, S. Fei, and Q.-L. Han, "Regional stabilization for discrete time-delay systems with actuator saturations via a delaydependent polytopic approach", IEEE Trans. Autom. Control, vol. 64, no. 3, pp. 1257-1264, Mar. 2019.

[3] D. Coutinho, C. E. de Souza, J. M. Gomes da Silva Jr., A. F. Caldeira, and C. Prieur, "Regional stabilization of input-delayed uncertain nonlinear polynomial systems", IEEE Trans. Autom. Control, vol. 65, no. 5, pp. 2300-2307, May 2020.

[4] J. M. Gomes da Silva Jr, and S. Tarbouriech, "Antiwindup design with guaranteed regions of stability: an LMI-based approach", IEEE Trans. Autom. Control, vol. 50, no. 1, pp. 106-111, Jan. 2005.

[5] E. Fridman, Introduction to time-delay systems: Analysis and control, Basel: Birkhäuser, 2014.

[6] E. Fridman, A. Pila, and U. Shaked, "Regional stabilization and $H_{\infty}$ control of time-delay systems with saturating actuators", Int. J. Robust Nonlinear Control, vol. 13, no. 9, pp. 885-907, 2003.

[7] K. Gu, V. L. Kharitonov, and J. Chen, Stability of time-delay systems, Boston: Birkhäuser, 2003.

[8] T. Hu, and Z. Lin, Control systems with actuator saturation: analysis and design, Boston: Birkhäuser, 2001.

[9] Y.-B. Huang, Y. He, J. An, and M. Wu, "Polynomial-type LyapunovKrasovskii functional and Jacobi-Bessel inequality: Further results on stability analysis of time-delay systems", IEEE Trans. Autom. Control, early access, Aug. 04, 2020, doi: 10.1109/TAC.2020.3013930.

[10] Y. Li, and Z. Lin, "Improvements to the linear differential inclusion approach to stability analysis of linear systems with saturated linear feedback", Automatica, vol. 49, pp. 821-828, 2013.

[11] Y. Li, and Z. Lin, "Stability and performance analysis of saturated systems via partitioning of the virtual input space", Automatica, vol. 53, pp. 85-93, 2015.

[12] Z. Lin, Low gain feedback, London: Springer-Verlag, 1999.

[13] Z. Lin, and H. Fang, "On asymptotic stabilizability of linear systems with delayed input", IEEE Trans. Autom. Control, vol. 52, no. 6, pp. 9981013, Jun. 2007.

[14] K. Liu, and E. Fridman, "Delay-dependent methods and the first delay interval", Syst. Control Lett., vol. 64, pp. 57-63, 2014.

[15] K. Liu, and E. Fridman, "Discrete-time network-based control under scheduling and actuator constraints", Int. J. Robust Nonlinear Control, vol. 25, no. 12, pp. 1816-1830, 2015.

[16] K. Liu, A. Seuret, "Comparison of bounding methods for stability analysis of systems with time-varying delays", J. Frankl. Inst., vol. 354, no. 7, pp. 2979-2993, 2017.

[17] L. Ma, Z. Wang, Y. Liu, and F. E. Alsaadi, "Distributed filtering for nonlinear time-delay systems over sensor networks subject to multiplicative link noises and switching topology", Int. J. Robust Nonlinear Control, vol. 29, no. 10, pp. 2941-2959, 2019.

[18] L. Ma, X. Fang, Y. Yuan, J. Zhang, and Y. Bo, "Dissipative control for nonlinear Markovian jump systems with mixed time-delays: The discrete-time case", Int. J. Robust Nonlinear Control, vol. 30, no. 7, pp. 2871-2888, 2020.

[19] P. Park, J. Ko, and C. Jeong, "Reciprocally convex approach to stability of systems with time-varying delays", Automatica, vol. 47, no. 1, pp. 235-238, 2011.

[20] A. Seuret, and J. M. Gomes Da Silva Jr, "Taking into account period variations and actuator saturation in sampled-data systems", Syst. Control Lett., vol. 61, no. 12, pp. 1286-1293, 2012.

[21] A. Seuret, and F. Gouaisbaut, "Wirtinger-based integral inequality: application to time-delay systems", Automatica, vol. 49, no. 9, pp. 28602866, 2013.

[22] S. Tarbouriech, G. Garcia, J. M. Gomes da Silva Jr, and I. Queinnec, Stability and stabilization of linear systems with saturating actuators, London: Springer-Verlag, 2011.
[23] Y. Wang, J. Xiong and D. W. C. Ho, "Globally optimal state-feedback LQG control for large-scale systems with communication delays and correlated subsystem process noises", IEEE Trans. Autom. Control, vol. 64, no. 10, pp. 4196-4201, Oct. 2019.

[24] Y.-Q. Wu, H. Su, and Z.-G. Wu, "Synchronisation control of dynamical networks subject to variable sampling and actuators saturation", IET Control Theory Appl., vol. 9, no. 3, pp. 381-391, Feb. 2015.

[25] X.-J. Xie and M. Jiang, "Dynamic state feedback stabilization of stochastic cascade nonlinear time-delay systems with SISS inverse dynamics", IEEE Trans. Autom. Control, vol. 64, no. 12, pp. 5132-5139, Dec. 2019.

[26] J. Xu, H. Zhang and L. Xie, "Consensusability of multiagent systems with delay and packet dropout under predictor-like protocols", IEEE Trans. Autom. Control, vol. 64, no. 8, pp. 3506-3513, Aug. 2019.

[27] S. Xu, G. Feng, Y. Zou, J. Huang, "Robust controller design of uncertain discrete time-delay systems with input saturation and disturbances", IEEE Trans. Autom. Control, vol. 57, no. 10, pp. 2604-2609, Oct. 2012.

[28] R. Yang, P. Shi, G.-P. Liu, and H. Gao, "Network-based feedback control for systems with mixed delays based on quantization and dropout compensation", Automatica, vol. 47, no. 12, pp. 2805-2809, 2011.

[29] D. Yue, Q.-L. Han, "Delayed feedback control of uncertain systems with time-varying input delay", Automatica, vol. 41, no. 2, pp. 233-240, 2005.

[30] C.-K. Zhang, F. Long, Y. He, et al., "A relaxed quadratic function negative-determination lemma and its application to time-delay systems", Automatica, vol. 113, Art. no. 108764, 2020.

[31] X.-M. Zhang, Q.-L. Han, A. Seuret, F. Gouaisbaut, and Y. He, "Overview of recent advances in stability of linear systems with time-varying delays", IET Control Theory Appl., vol. 13, no. 1, pp. 1-16, Jan. 2019.

[32] B. Zhou, Z. Lin, and G. Duan, "A parametric Lyapunov equation approach to the design of low gain feedback", IEEE Trans. Autom. Control, vol. 53, no. 6, pp. 1548-1554, Jun. 2008.

[33] B. Zhou, Z. Lin, and G.-R. Duan, "Global and semi-global stabilization of linear systems with multiple delays and saturations in the input", SIAM J. Control Optim., vol. 48, no. 8, pp. 5294-5332, 2010.

[34] B. Zhou, Z.-Y. Li, and Z. Lin, "Stabilization of discrete-time systems with multiple actuator delays and saturations", IEEE Trans. Circuits Syst.-I, vol. 60, no. 2, pp. 389-400, Feb. 2013.

[35] S. Y. Yoon, and Z. Lin, "Truncated predictor feedback control for exponentially unstable linear systems with time-varying input delay", Syst. Control Lett., vol. 62, no. 10, pp. 837-844, 2013. 